\section{Diagnostic Value of Direct Examination of Protected Specimen Brush Samples in Nosocomial Pneumonia}

\author{
A. H. Mertens ${ }^{1 *}$, J. M. Nagler ${ }^{2}$, \\ D. I. Galdermans ${ }^{3}$, H. R. Slabbynck ${ }^{3}$, \\ B. S. Weise ${ }^{1}$, D. Coolen ${ }^{3}$
}

The value of direct examination of Giemsa and Gram stains of cytospin preparations of protected specimen brush samples was compared to that of quantitative culture. Sixty-one samples from patients suspected to have nosocomial pneumonia were analysed. Twenty-five samples were positive by quantitative culture, 21 of which contained microorganisms seen by direct examination. The presence of leucocytes was not specific for a positive culture, but in their absence, a positive culture was unlikely. The presence of intracellular organisms always correlated with a positive culture, but was not very sensitive.

The protected specimen brush (PSB) technique combined with quantitative culture (cutoff, $10^{3} \mathrm{cfu} / \mathrm{ml}$ ) has been recommended as a reference method for the diagnosis of ventilator-associated nosocomial pneumonia (1). Quantitative cultures of PSB samples require overnight incubation to obtain results. This delay can be overcome by direct microscopic examination of the samples.

Examination of bronchoalveolar lavage fluid provides reliable information on the percentage of inflammatory cells with intracellular organisms (ICOs) present, and this correlates well with the diagnosis of pneumonia (2-4). The importance of the presence of intracellular organisms in PSB cytospin preparations, however, has not yet been studied.

We compared the diagnostic value of direct examination of cytospin preparations of PSB samples

${ }^{1}$ Laboratory for Clinical Microbiology, ${ }^{2}$ Department of Intensive Care, and ${ }^{3}$ Department of Pneumology, Middelheim General Hospital, Lindendreef 1, B-2020 Antwerp, Belgium. to that of quantitative culture. The relevance of both the cellular composition of the samples and the presence of bacteria and intracellular organisms in the samples was investigated.

Materials and Methods. From March 1992 to August 1994, all patients suspected to have nosocomial pneumonia who were admitted to the medical intensive care unit and the Department of Pneumology of the Middelheim General Hospital were evaluated. Nosocomial pneumonia was diagnosed on the basis of a new pulmonary infiltrate on chest radiograph $48 \mathrm{~h}$ after admission, in association with fever $\left(>38^{\circ} \mathrm{C}\right)$ or elevated leucocytosis. In patients ventilated for more than $48 \mathrm{~h}$, additional criteria used to define pneumonia were worsening hypoxia and/or increased purulent tracheal secretions detected by visual inspection. Sixty-one patients ( 38 men, 23 women) with a mean age of 59 years were included in the study. Forty-two $(69 \%)$ were mechanically ventilated, and $39(64 \%)$ had received antibiotics prior to sampling for a mean duration of 4.5 days. Sixteen suffered from neurological disorders and nine had a neoplastic condition, whereas the other 27 had various underlying diseases or conditions (chronic lung disease, abdominal surgery, polytrauma, diabetes mellitus).

As described by Marquette et al. (5), a single catheter (Novatech 13127; France) with a double port on its proximal tip was used for PSB sampling. After sampling, the brush was advanced beyond the sheath and vigorously vortexed in $1 \mathrm{ml}$ of sterile Ringer's solution for at least 60 seconds. Quantitative culture was performed by the calibrated loop method. Ten $\mu$ l of the Ringer's lactate solution was inoculated onto Columbia agar with $5 \%$ sheep blood (Becton-Dickinson, France); Centers for Disease Control anaerobe blood agar; MacConkey agar; and Sabouraud dextrose agar with chloramphenicol. One hundred $\mu 1$ was inoculated with a calibrated pipette onto chocolate II agar (BectonDickinson) and buffered charcoal-yeast extract agar $(\mathrm{BCYE} \alpha)$. Plates were incubated at $36^{\circ} \mathrm{C}$ under adequate aerobic and anaerobic conditions, and were evaluated for growth after $24 \mathrm{~h}$, $48 \mathrm{~h}$, and five days. Numbers of bacteria in the original fluid were estimated by colony counts of each morphotype and expressed in colonyforming units per millilitre $(\mathrm{cfu} / \mathrm{ml})$. A count of $\geq 10^{3} \mathrm{cfu} / \mathrm{ml}$ was used as the cutoff point for determining a positive culture. Cultures were performed according to standard procedures. 
Table 1: Organisms detected by culture or positive direct examination of 27 protected specimen brush samples.

\begin{tabular}{|c|c|c|c|c|c|}
\hline $\begin{array}{l}\text { Sample } \\
\text { no. }\end{array}$ & $\begin{array}{l}\text { Organism(s) } \\
\text { cultured }\end{array}$ & $\begin{array}{l}\text { Colony } \\
\text { count } \\
(\mathrm{cfu} / \mathrm{ml})\end{array}$ & $\begin{array}{c}\text { Organisms } \\
\text { detected by } \\
\text { direct examination }\end{array}$ & $\begin{array}{l}\text { Presence } \\
\text { of PMNs }\end{array}$ & $\begin{array}{l}\text { Presence } \\
\text { of ICOs }\end{array}$ \\
\hline 1 & Escherichia coli, Enterobacter cloacae & $10^{3}, 10^{3}$ & g.n. bacilli & yes & yes \\
\hline 2 & Pseudomonas aeruginosa & $>10^{5}$ & g.n. bacilli & yes & yes \\
\hline 3 & Klebsiella pneumoniae, Enterobacter cloacae & $4 \times 10^{3}, 5 \times 10^{3}$ & none & yes & no \\
\hline 4 & Pseudomonas aeruginosa & $2 \times 10^{4}$ & g.n. bacilli & yes & no \\
\hline 5 & Haemophilus influenzae, Lactobacillus acidophilus & $10^{3}, 7 \times 10^{3}$ & g.p.bacilli, g.p. cocci & yes & yes \\
\hline 6 & Haemophilus influenzae & $2 \times 10^{3}$ & g.n. bacilli & yes & no \\
\hline 7 & Staphylococcus aureus, Streptococcus milleri & $3 \times 10^{3}, 10^{3}$ & g.p. cocci & yes & yes \\
\hline 8 & Enteroccocus faecalis & $2 \times 10^{3}$ & none & yes & no \\
\hline 9 & Citrobacter diversus, Neisseria mucosa & $>10^{5},>10^{5}$ & g.n. bacilli, g.p. cocci & yes & yes \\
\hline 10 & Staphylococcus aureus & $10^{3}$ & g.p. cocci & yes & yes \\
\hline 11 & Escherichia coli & $10^{5}$ & g.n. bacilli & yes & no \\
\hline 12 & Pseudomonas aeruginosa & $6 \times 10^{3}$ & g.n. bacilli & yes & yes \\
\hline 13 & $\begin{array}{l}\text { Escherichia coli, Morganella morganii, } \\
\text { Bacteroides thetaiotaomicron, Bacteroides fragilis, } \\
\text { Bacteroides uniformis }\end{array}$ & $\begin{array}{c}3 \times 10^{3}, 3 \times 10^{3} \\
3 \times 10^{3}\end{array}$ & none & no & no \\
\hline 14 & Pseudomonas aeruginosa & $10^{5}$ & g.n. bacilli & yes & yes \\
\hline 15 & Neisseria subflava, $\alpha$-haemolytic streptococci & $4 \times 10^{3}, 10^{4}$ & $\begin{array}{c}\text { g.n. cocci, g.p. cocci, } \\
\text { g.n. bacilli }\end{array}$ & yes & no \\
\hline 16 & Pseudomonas aeruginosa & $>10^{5}$ & g.n. bacilli & yes & no \\
\hline 17 & Pseudomonas aeruginosa & $10^{5}$ & g.n. bacilli, g.p. cocci & yes & no \\
\hline 18 & Pseudomonas aeruginosa & $>10^{5}$ & g.n. bacilli & yes & no \\
\hline 19 & $\alpha$-haemolytic streptococci & $2 \times 10^{3}$ & none & yes & no \\
\hline 20 & Acinetobacter baumannii, $\alpha$-haemolytic streptococci & $3 \times 10^{3}, 3 \times 10^{3}$ & $\begin{array}{c}\text { g.n. bacilli, g.p. cocci, } \\
\text { g.p. bacilli }\end{array}$ & yes & yes \\
\hline 21 & Klebsiella pneumoniae & $5 \times 10^{4}$ & g.n. bacilli & yes & no \\
\hline 22 & Escherichia coli & $>10^{5}$ & g.n. bacilli & yes & no \\
\hline 23 & Streptococcus pneumoniae & $>10^{5}$ & g.p. cocci & yes & yes \\
\hline 24 & Pseudomonas aeruginosa & $10^{5}$ & g.n. bacilli & yes & yes \\
\hline 25 & Escherichia coli & $10^{5}$ & g.n. bacilli & yes & yes \\
\hline 26 & none & & g.n. bacilli & yes & no \\
\hline 27 & none & & yeasts & no & no \\
\hline
\end{tabular}

g.n., gram-negative; g.p., gram-positive; ICO, intracellular organisms; PMN, polymorphonuclear leucocytes.

For direct microscopic analysis, two slides were prepared by cytocentrifugation using a Shandon Cytospin 2 (Southern Product, UK). One MayGrünwald-Giemsa stain and one Gram stain were made, using $140 \mu \mathrm{l}$ of fluid for each slide. At least 20 high-power fields were examined. In $70 \%$ of the cases, microscopic examination was performed on the day of sampling before the results of culture were known. The presence of microorganisms was assessed on the stains. Morphology and staining characteristics of organisms seen on Gram stain were recorded. Direct examination for the presence of microorganisms was considered truly positive when at least one morphotype was seen and subsequently cultured $\left(>10^{3} \mathrm{cfu} / \mathrm{ml}\right.$ ). The result was considered falsely positive when microorganisms were seen but not cultured in sufficient number. Polymorphonuclear leucocytes (PMNs) were considered present when at least one cell per field was observed. To determine the percentage of PMNs, other inflammatory cells and bronchial cells - but not erythrocytes - were considered. Finally, the presence of intracellular organisms was investigated. If microorganisms were seen, the entire preparation was screened for intracellular organisms. At least three intracellular organisms had to be recognised without a doubt on either the Gram or the Giemsa stain. Samples containing $1 \%$ or more squamous epithelial cells were not evaluated. The unpaired Student's test was used to compare the means of two groups. A p-value of $<0.05$ was considered significant.

Results and Discussion. Table 1 shows the results of culture and direct examination of 61 PSB specimens. Twenty-five samples were positive by quantitative culture, 15 of which were obtained from patients with previous antibiotic treatment, and ten from patients with no previous antibiotic therapy.

Table 2 shows the correlations between the presence of microorganisms, PMNs, and intracellular organisms detected by direct examination and 
the results of PSB culture. The sensitivity of the presence of microorganisms compared to culture was $84 \%$ and the specificity $94.4 \%$; the positive predictive value was $91.3 \%$ and the negative predictive value $89.4 \%$.

The sensitivity of the presence of PMNs compared to positive culture was $96 \%$, the specificity $33.3 \%$, the positive predictive value $50 \%$, and the negative predictive value $92.3 \%$. A differential cell count was performed for 48 samples. The percentage of PMNs was significantly higher in culturepositive samples $(59 \% \pm 29)$ than in culturenegative samples $(18.5 \% \pm 24)(\mathrm{p}<0.001)$; however, the range was very broad. Differential cell count does not seem to offer substantial diagnostic advantages. Although PMNs were frequently present in the preparations of culture-positive samples, they were also present in a large number of culture-negative samples. On the other hand, the probability of a positive culture was very low in the absence of PMNs.

The sensitivity of the presence of intracellular organisms was $44 \%$, the specificity $100 \%$, the positive predictive value $100 \%$, and the negative predictive value $72 \%$. Initial studies $(6,7)$, which used slides with one drop of fluid directly placed onto each glass slide, showed a low sensitivity for Gram stain of PSB samples. Direct smear of the brush on the slide before washing in Ringer's lactate enhances the sensitivity of the direct examination (8), although this procedure risks contamination of the sample and means a loss of material for culture, unless two separate brushes are used (9). Cytocentrifugation is an effective method for increasing the number of cells and microorganisms in preparations for direct examination of body fluids (10).

Marquette et al. (11) obtained satisfactory results from microscopic examination of cytospin preparation of PSB samples. Our results are comparable with those of Marquette et al. (11), although we used a slightly different definition for the presence of microorganisms.

Recent data have shown that microscopic detection of intracellular organisms recovered by bronchoalveolar lavage is a specific method for early and rapid diagnosis of pneumonia in mechanically ventilated patients $(7,2-4,12)$. The cutoff point for a significant percentage of intracellular organisms present in bronchoalveolar lavage smears has been largely debated $(2,3$, 12-14).
Table 2: Correlation between presence of microorganisms, presence of polymorphonuclear leukocytes (PMNs), presence of inflammatory cells containing intracellular organisms (ICOs), and culture of 61 protected specimen brush samples from patients suspected to have nosocomial pneumonia.

\begin{tabular}{lrc}
\hline & \multicolumn{2}{c}{ Results of culture } \\
\cline { 2 - 3 } & Positive & Negative \\
\hline Microorganisms present & 21 & 2 \\
Microorganisms absent & 4 & 34 \\
PMNs present & 24 & 24 \\
PMNs absent & 1 & 12 \\
ICOs present & 11 & 0 \\
ICOs absent & 14 & 36 \\
& & \\
\hline
\end{tabular}

Our study shows that intracellular organisms may be recognised in PSB specimens. However, as the cells are sometimes distorted (probably due to the brushing procedure), they are more difficult to identify, and quantification may be problematic. We, therefore, determined that three intracellular organisms had to be recognised without a doubt upon direct examination.

In conclusion, this study demonstrates that cytospin preparations are well suited for microscopic examination of PSB samples. The presence of microorganisms in Gram or Giemsa stains predicts fairly well the results of quantitative bacterial culture. In the absence of PMNs, a positive quantitative culture is unlikely. The presence of inflammatory cells containing intracellular organisms always corresponded with a positive culture in our study.

\section{References}

1. Chastre J, Viau F, Brun $P$, Pierre J, Dauge MC, Bouchama A, Akesbi A, Gibert C: Prospective evaluation of the protected specimen brush for the diagnosis of pulmonary infections in ventilated patients. American Review of Respiratory Disease 1984, 130: 924-929.

2. Chastre J, Fagon JY, Soler $P$, Bornet $M$, Domart $Y$, Trouillet JL, Gibert C, Hance AJ: Diagnosis of nosocomial pneumonia in intubated patients undergoing ventilation: comparison of usefulness of bronchoalveolar lavage and protected specimen brush. American Journal of Medicine 1988, 85: 499-506.

3. Chastre J, Fagon JY, Soler P, Domart Y, Pierre J, Dombret MC, Gibert C, Hance AJ: Quantification of BAL cells containing intracellular bacteria rapidly identifies ventilated patients with nosocomial pneumonia. Chest 1989, 95, Supplement 2: 190-192. 
4. Chastre J, Fagon JY, Bornet-Lecso M, Calvat S, Dombret MC, Khani RA, Basset F, Gibert C: Evaluation of bronchoscopic techniques for the diagnosis of nosocomial pneumonia. American Journal of Respiratory and Critical Care Medicine 1995, 152: 231-240.

5. Marquette $\mathrm{CH}$, Ramon $\mathrm{P}$, Courcol R, Wallaert B, Tonnel $A B$, Voisin $C$ : Bronchoscopic protected catheter brush for the diagnosis of pulmonary infections. Chest 1988, 93: 746-750.

6. Higuchi JG, Coolson JJ, Johanson WG: Bacteriologic diagnosis of nosocomial pneumonia in primates. American Review of Respiratory Disease 1982, 125: 53-57.

7. Meduri GU, Beals DH, Maijub AG, Baselski V: Protected bronchoalveolar lavage: a new bronchoscopic technique to retrieve uncontaminated distal airway secretions. American Review of Respiratory Disease 1991, 143: 855-864.

8. Poilock HM, Hawkins EL, Bonner JR, Sparkman T, Bass JB: Diagnosis of bacterial pulmonary infections with quantitative protected catheter cultures obtained during bronchoscopy. Journal of Clinical Microbiology 1983, 17 : 255-259.

9. Teaque RB, Wallace RJ, Awe RJ: The use of quantitative sterile brush culture and Gram stain analysis in the diagnosis of lower respiratory tract infection. Chest 1981, 79: 157-161.

10. Chapin-Robertson K, Dahlberg SE, Edberg SC: Clinical and laboratory analyses of cytospin-prepared Gram stains for recovery and diagnosis of bacteria from sterile body fluids. Journal of Clinical Microbiology 1992, 30: 377-380.

11. Marquette $\mathrm{CH}$, Wallet $F$, Nevière $R$, Copin $M C$, Saulnier F, Drault JN, Mehdaoui H, Mathieu D, Ramon P: Diagnostic value of direct examination of the protected specimen brush in ventilator-associated pneumonia. European Respiratory Journal 1994, 7: 105-113.

12. Meduri GU, Belenchia JM, Estes RJ, Wunderink RG, El Torky M, Leeper KV: Fibroproliferative phase of ARDS: clinical findings and effects of corticoids. Chest 1991, 100: 943-952.

13. Solé-Violan J, Rodrigez de Castro F, Rey A, MartinGonzalez J, Cabrero-Navarro P: Usefulness of microscopic examination of intracellular organisms in lavage fluid in ventilator-associated pneumonia. Chest 1994, 106: 889-894.

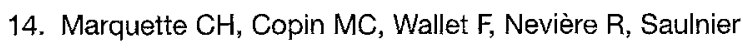
F, Mathieu D, Durocher A, Ramon P, Tonnel AB: Diagnostic tests for pneumonia in ventilated patients: prospective evaluation of diagnostic accuracy using histology as a diagnostic gold standard. American Journal of Respiratory and Critical Care Medicine 1995, 151: 1878-1888.

\section{Urine Samples as a Possible Alternative to Serum for Human Immunodeficiency Virus Antibody Screening}

\author{
P. Martínez*, R. Ortiz de Lejarazu, \\ J. M. Eiros, J. De Benito, \\ A. Rodríguez-Torres
}

The detection of specific antibodies against human immunodeficiency virus (HIV) was tested by dot blot enzyme immunoassay in 95 urine samples from 72 individuals infected with HIV and 23 seronegative individuals. Western blot of paired serum samples from these same individuals was used as the gold standard. The dot blot tested had a sensitivity of $97.2 \%$ and a specificity of $100 \%$; only two samples from HIV-infected individuals at Centers for Disease Control (CDC) stages II and IV were non-reactive. Reactive and discrepant samples (serum/urine) were confirmed by Western blot, which had a sensitivity of $98.6 \%$ and a specificity of $100 \%$. The most commonly observed Western blot reactivity pattern in urine samples included bands against three groups of HIV structural proteins (ENV, POL, and GAG). The results indicate that urine can be used in screening for HIV antibodies in epidemiological studies of high-prevalence populations, though it is not recommended for individualized diagnostic purposes.

The increased demand for the capability to detect antibodies against human immunodeficiency virus (HIV) in different situations (diagnosis, epidemiological studies, etc.) calls for easily performed tests that enable large numbers of samples to be processed without requiring specialized equipment or personnel. It would also be desirable if such a test used a type of sample that is both easy to obtain and reliable as regards diagnostic results. Such a test could serve as a viable alternative to serological tests currently used in the diagnosis of

Microbiology Laboratory Service, University Hospital, c/ Ramón y Cajal s. n., 47011 Valladolid, Spain. 